\title{
In memoriam Christian Hess (1950-2019)
}

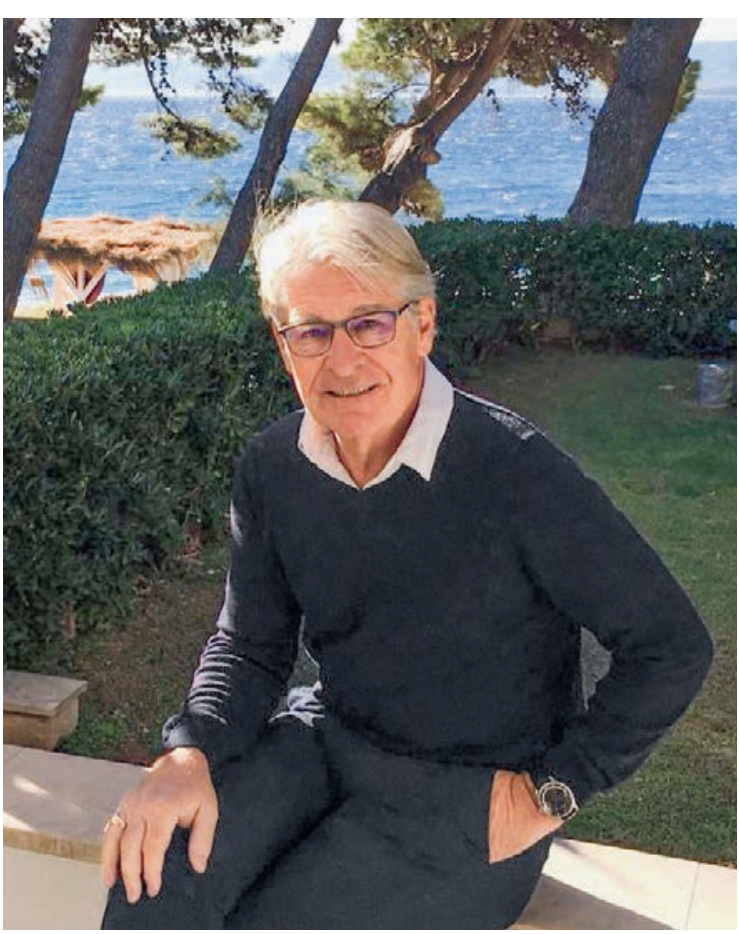

Christian Hess, 10.8.1950-25.9.2019

Es gibt Menschen, die in der direkten Begegnung so präsent und lebendig wirken, dass die Nachricht von ihrem Tod kaum fassbar ist. In meiner Wahrnehmung traf dies auf Christian Hess in höchstem Masse zu. Bei unserer letzten Begegnung im vergangenen Sommer war er voller Leben, trotz seiner Krankheit, trotz der Belastung durch die Chemotherapie, herzlich, humorvoll, geistreich, positiv. Dass sein Schicksal auf Messers Schneide stand, hatte der herausragende Arzt und Mediziner glasklar vor Augen. Er verdrängte diese Tatsache keineswegs, sprach sie im Gegenteil im persönlichen Gespräch unverblümt an.

Die Dinge beim Namen zu nennen, selbst wenn dies unangenehm war und Widerstand provozierte, dabei aber die Leichtigkeit des Seins nicht zu verlieren: Auch das zeichnete Christian Hess aus. Wir lernten uns $2010 \mathrm{im}$ Zusammenhang mit seinem Engagement gegen die Einführung des Fallpauschalensystems SwissDRG kennen. Gemeinsam mit seinem Freund und Chefarztkollegen Urs Strebel hatte er in der SÄZ einen vielbeachteten Artikel verfasst, in dem die beiden Autoren zu einem DRGMoratorium aufriefen. Es beeindruckte ihn, dass es möglich war, im Organ der Schweizer Ärztinnen und
Ärzte an prominenter Stelle eine Stellungnahme zu publizieren, die der offiziellen Haltung der FMH zuwiderlief. Spontan lud er mich ein, das «Modell SÄZ» - offizielles Organ und redaktionell unabhängige Zeitschrift -im Rahmen der wöchentlichen Hausarztfortbildung im Spital Affoltern vorzustellen, wo er seit 1988 als Chefarzt Innere Medizin und seit 1999 als Ärztlicher Leiter tätig war. Beim anschliessenden Essen fiel mir zum ersten Mal sein lausbubenhaftes Lachen auf, das bei ihm in unverwechselbarer Weise mit einem energischen Zug gepaart war. Man lachte in seiner Gegenwart viel, auch wenn die Themen, über die man sprach, oft nicht zum Lachen waren.

Dass in seiner Persönlichkeit scheinbar Gegensätzliches in gewinnender Weise zusammenfand, war vielleicht das wesentliche Erfolgsgeheimnis von Christian Hess. Lockerheit gepaart mit Willensstärke, intellektuelle Brillanz ohne Abgehobenheit, Hartnäckigkeit, die frei von Verbissenheit war - die Kombination solcher Eigenschaften findet sich selten in einer Person. Selbstverständlich muss er wie jeder Mensch auch Schwächen gehabt haben. Aus eigener Erfahrung kann ich dazu aber nichts sagen, dafür hätte es wahrscheinlich eines intensiveren Kontaktes bedurft. Was ich dagegen erlebte, war ein genuines Interesse am jeweiligen $\mathrm{Ge}$ genüber und eine Jugendlichkeit, die bei ihm auch im siebten Lebensjahrzehnt nie künstlich wirkte. «Still going strong?», begrüsste er mich einmal, nachdem wir uns länger nicht gesehen hatten. Bei vielen Mittsechzigern hätte ein solcher Spruch aufgesetzt gewirkt. Bei ihm passte er.

Man lachte in seiner Gegenwart viel, auch wenn die Themen, über die man sprach, oft nicht zum Lachen waren.

Diese Eigenschaften bildeten die Basis für ein vielfältiges, erfolgreiches ärztliches Wirken, aber auch für ein erfülltes Familienleben. Die Anlagen dafür wurden ihm in die Wiege gelegt, doch sie mussten erst reifen. Als Kind eher schüchtern, sollen ihm Kindergarten und Schule zu Beginn Bauchschmerzen bereitet haben. In den Ferien bei seinem Onkel litt Christian unter Heimweh. Zur Ablenkung nahm ihn der Arzt auf seine abendlichen Hausbesuche mit. Dies beeindruckte den Jungen so sehr, dass er fortan selbst Arzt werden wollte. Diesem Entschluss blieb er treu, als aus dem scheuen 
Knaben ein langhaariger Jüngling in engen Jeans geworden war, der die Trommelfelle seiner Familie beim Mittagessen mit dem Rolling-Stones-Song «Satisfaction" strapazierte. Auch das Herz seiner späteren Frau Annina eroberte er nicht mit Schüchternheit, sondern mit einem Schuss spitzbübischer Frechheit: Ausgerechnet in der Wohnung ihres damaligen Freundes wagte er in der Silvesternacht 1971/72 den ersten - und langfristig entscheidenden - Kuss.

Christian Hess' ärztliches Wirken ist ohne die Verbindung mit seiner Frau nicht denkbar. Eben Mutter geworden, begleitete sie ihn 1981 nach Tansania, wo er während zweier Jahre am St.-Francis-Spital in Ifakara tätig war. Dort wurde das zweite von drei Kindern der Familie Hess-Cabalzar geboren. Als er nach der Rückkehr mit 38 Jahren zum jüngsten Chefarzt im Kanton Zürich berufen wurde, sagte die klinische Psychotherapeutin "Ja» zur klassischen Rollenverteilung. Doch dabei blieb es nicht. Sowohl im Spital Affoltern, wo sie die Psychotherapie leitete und entscheidend prägte, als auch in der von ihr initiierten "Akademie Menschenmedizin" bildete sie mit Ehemann ein erfolgreiches «Power Couple», wie es ein Freund umschrieb. Konstellationen, in denen Partnerschaften privat und beruflich funktionieren müssen, sind bekanntlich riskant. Schon manche Beziehung ist daran zerbrochen. Christian und Annina HessCabalzar schafften es, ein einzigartiges Spitalmodell darauf aufzubauen.

Die Frage, wie eine würdige Medizin praktiziert werden kann, beschäftigte Christian Hess zeitlebens.

Die Rede ist vom "Modell Affoltern", das die beiden zwar nicht im Alleingang entwickelten, zu dem sie aber entscheidende Impulse gaben und zu dessen "Aushängeschildern» sie - vielleicht nicht ganz freiwillig - wurden. Den Grundstein zu diesem Modell hatte schon Hess' früh verstorbener Chefarzt-Vorgänger Peider Mohr gelegt. Wie der langjährige Direktor des Spitals Affoltern Ruedi Wegmann in seinem Nachruf auf Christian Hess schrieb, steht das Modell Affoltern «einerseits für die optimale Vernetzung zwischen Spital, Hausärzten, Spitexdiensten und Psychotherapeuten. Es beinhaltet aber auch eine andere Form von Krankheitsverständnis.»

Die Frage, wie eine würdige Medizin praktiziert werden kann und welches Menschenbild und Krankheitsverständnis ihr zugrunde liegen sollte, beschäftigte Christian Hess zeitlebens. Seine Reflexionen und Erkenntnisse legte er im 2006 erschienenen Buch «Menschenmedizin» vor, das er gemeinsam mit seiner Frau verfasste. Dieser Begriff steht für eine Medizin, die sich dem Menschen in einem umfassenden Sinn zuwendet. Sie ist auf einen individuellen, interprofessionellen medizinischen Behandlungsprozess ausgerichtet, der Erkenntnisse aus Psychotherapie, Philosophie, Ethik und Kunst einbezieht. An diesem Medizinverständnis orientierte sich Christian Hess in seiner ärztlichen Tätigkeit und in seinem Umgang mit Patienten und Mitarbeitenden. Bereits 1998 hatte er im Spital Affoltern Philosophie-Wochen eingeführt, die eine grosse, weit über die Region ausstrahlende Resonanz erzeugten. Die Integration von Palliative Care und Psychiatrie in den Grundversorgungsauftrag des Spitals trieb er schon zu einem Zeitpunkt voran, als solche Konzepte noch Neuland waren.

Langjähriges Mitglied der Ethikkommission des Kantons Zürich, Lehrbeauftragter an der Universität Zürich, Kommissionsmitglied in Arbeitsgruppen der Schweizerischen Akademie der Medizinischen Wissenschaften, Vorstandsmitglied der Ärztegesellschaft Zürich, aktiv in unzähligen Projekten und Organisationen des Gesundheitswesens: Es ist müssig, im Rahmen dieses Nachrufs die Engagements von Christian Hess im medizinischen, ethischen und gesundheitspolitischen Bereich erschöpfend aufzählen zu wollen.

Nicht fehlen darf aber seine tragende Rolle als Vorstandsmitglied der Akademie Menschenmedizin. Dieser Verein, der 2009 auf Initiative seiner Frau Annina gegründet wurde, setzt sich mit Projekten, Dienstleistungen, Symposien und weiteren Aktivitäten für ein Gesundheitswesen ein, «das den Menschen und nicht die Kosten im Blick hat und trotzdem bezahlbar ist».* Christian Hess hat der Akademie viel gegeben. Mit seinem Tod ist sie zu seinem Vermächtnis geworden.

Arzt, Lehrer, Autor, Gesundheits- und Standespolitiker, Organisator und Führungspersönlichkeit, Familienvater und Ehemann, Freund - Christian Hess hat in seinen 69 Lebensjahren so viel vollbracht, dass es eigentlich für mehrere Leben reichen würde. Dennoch überwiegt bei seinen Weggefährten sicher der Eindruck, dass er mitten aus dem Leben gerissen wurde und viel zu früh gehen musste. Die Impulse, die er gesetzt hat, werden indessen weiterwirken, und es bleibt die Erinnerung an einen grossartigen Menschen. Ich bin mir ziemlich sicher, dass sich Christian darüber freuen würde, wenn in diesem Nachruf die Rockband «Steppenwolf» mit dem Titel eines ihrer Alben aus den 70ern das letzte Wort hätte. Und so sei es denn auch: Rest in Peace, lieber Christian.

Dr. med. et lic. phil. Bruno Kesseli, Chefredaktor SÄZ 2005-7/2019 